\title{
Terapia nutricional enteral em pacientes oncológicos sob cuidados paliativos: uma análise da bioética
}

\section{Enteral nutrition therapy in cancer patients under palliative care: a bioethics analysis}

\author{
Viviane Campos Leite Loyolla', Leocir Pessini², Andrea Bottoni ${ }^{3}$, \\ Sandra Caires Serrano ${ }^{4}$, Ana Lucia Teodoro ${ }^{5}$, Adriana Bottoni ${ }^{6}$
}

\begin{abstract}
Loyolla VCL, Pessino L, Bottoni A, Serrano SC, Teodoro AL, Bottoni A. Terapia nutricional enteral em pacientes oncológicos sob cuidados paliativos: uma análise da bioética. Saúde, Ética \& Justiça. 2011;16(1):47-59.

RESUMO: A terapia nutricional em pacientes com doenças muito avançadas ou em doentes considerados em período de cuidados terminais está sendo frequentemente discutida pelos profissionais de saúde. O comprometimento do estado nutricional é uma complicação presente em pacientes com câncer, em que a perda de peso frequentemente é o primeiro sintoma ocorrido e está associado ao aumento da morbi-mortalidade. Em cuidados paliativos, a terapia nutricional deve ter como primeiro objetivo aumentar a qualidade de vida do paciente, minimizando os sintomas relacionados à nutrição e adiar ou suspender a perda da autonomia. Sendo a bioética um instrumento de reflexão e ação, seus princípios e referenciais devem estar presentes em cada atitude e decisão, havendo sempre a necessidade de uma reflexão profunda da equipe, juntamente com a família e o paciente, priorizando a beneficência do paciente. $O$ trabalho teve como objetivo principal analisar, sob o ponto de vista da Bioética, a utilização da terapia nutricional enteral em pacientes oncológicos sob cuidados paliativos, verificando a participação dos pacientes e de seus familiares na decisão sobre a utilização dessa terapia e conhecendo sua visão a respeito dela.
\end{abstract}

DESCRITORES: Bioética; Neoplasias; Cuidados paliativos; Terapia nutricional.

\footnotetext{
1. Nutricionista, Mestre em Bioética pelo Centro Universitário São Camilo, Especialista em Nutrição Clínica pelo Centro Universitário São Camilo.

2. Professor Doutor em Bioética e Teologia Moral, Presidente Provincial das Entidades Camilianas do Brasil.

3. Professor Doutor do Curso de Medicina da Universidade de Mogi das Cruzes.

4. Médica Responsável pelo Serviço de Cuidados Paliativos do Hospital A. C. Camargo.

5. Enfermeira Responsável pelo Serviço de Cuidados Paliativos do Hospital A. C. Camargo.

6. Gerente Médica do Hospital A. C. Camargo.
}

Endereço para correspondência: Rua Domiciano Rossi 600 apto 54H - Chácara Inglesa - S. Bernardo do Campo, SP. CEP: 09726-121. e-mail: vloyolla@ hotmail.com 
Loyolla VCL, et al. Terapia nutricional enteral em pacientes oncológicos sob cuidados paliativos.

\section{INTRODUÇÃO}

A discussão sobre ética tem sido retomada e ampliada nas últimas décadas, nos diversos espaços da sociedade, particularmente no campo da saúde ${ }^{1}$.

A maioria das sociedades nas quais 0 atendimento à saúde é exercido com os cuidados e com a exigência que o desenvolvimento técnico e científico impõe tem se preocupado em definir a competência dos profissionais de saúde, com diferentes habilidades, para resolver as complexas situações clínicas que afetam o paciente ${ }^{2}$.

Atualmente, para que o profissional seja reconhecido como competente, é necessário que tenha conhecimentos mais amplos, além dos tradicionais. Não basta ser exímio tecnicamente, é imprescindível que esteja em constante atualização também sobre os aspectos éticos e legais, devendo nortear suas condutas a partir deles, posicionandose de forma crítica e reflexiva diante dos dilemas éticos e morais que permeiam seu cotidiano ${ }^{3}$.

O profissional da saúde deve sempre promover, em primeiro lugar, o bem-estar do paciente, evitando danos, tratamentos inúteis $\mathrm{e}$ desnecessários. Deve dominar conhecimentos, saberes, técnicas e habilidades, além de demonstrar compaixão, para compreender como a pessoa de quem cuida sente a experiência do processo saúdedoença e quais seus valores e crenças ${ }^{3}$.

Como afirmam Pessini e Barchifontaine ${ }^{4}$, é um desafio permanente para os profissionais da saúde juntar à crescente aquisição do conhecimento científico a sabedoria humana que "(...) vai descobrir como companheiras inseparáveis a ousadia científica de inovar, de um lado, e prudência ética do mundo dos valores humanos, de outro".

A Bioética, ou ética da vida, é um instrumento de reflexão e ação, um espaço de diálogo transprofissional, transdisciplinar e transcultural na área da saúde e da vida, um grito pelo resgate da dignidade da pessoa humana, dando ênfase à qualidade de vida pautada na tolerância e na solidariedade ${ }^{5}$. Segundo Potter ${ }^{6}$, é considerada uma ponte entre a ciência e a ética, proporcionando a interação entre o conhecimento biológico, a ciência dos seres vivos e o conhecimento dos valores humanos.

Tendo como base a Bioética, podemos refletir sobre questões relacionadas a diversos campos. Como agir no que se refere à nutrição e à hidratação em casos de pacientes terminais?

O questionamento sobre a terapia nutricional em pacientes com doenças muito avançadas, ou em doentes que são considerados em período terminal, é levantado por McKinlay em 2004, já que muitos desses pacientes encontram-se gravemente desnutridos nesse momento.

A terapia nutricional deve ser indicada em todos os casos de pacientes sob cuidados paliativos?

Cuidados Paliativos, segundo a Organização Mundial da Saúde ${ }^{8}$, são descritos como cuidados direcionados a pacientes com doenças progressivas avançadas, que requerem a atuação de uma equipe multidisciplinar apropriada. O principal objetivo do tratamento terapêutico é proporcionar uma melhor condição de vida para o paciente e para seus familiares, considerando seus aspectos psicológico, social e espiritual, e administrar os sintomas da doença.

Para pacientes em cuidados paliativos, a nutrição não é somente fornecimento de energia, mas parte do tratamento. O estado nutricional precário gera condições para que a doença progrida, aumenta o risco de complicações e reduz a eficiência do tratamento. Portanto, a alimentação tem um lugar central na vida de todas as pessoas, um papel fundamental para o controle do avanço de uma doença progressiva ${ }^{8}$.

Em pacientes com câncer, os problemas nutricionais, a anorexia e a subsequente perda de peso são amplamente frequentes e podem ocorrer por diversas razões, devido ao próprio processo da doença, aos tratamentos e ao resultado de distúrbios do metabolismo ou sintomas de depressão ${ }^{8}$. Em 80\% dos pacientes com câncer em estágio avançado, particularmente em portadores de câncer de cabeça e pescoço, gastrintestinal e câncer de pulmão, são observados aumento da morbidade e mortalidade e redução da qualidade de vida, estando associados a um prognóstico desfavorável ${ }^{9,10}$.

A nutrição enteral é uma técnica terapêutica amplamente utilizada para aportar nutrientes de forma efetiva aos pacientes que têm incapacidade de receber seus requerimentos nutricionais por via oral. Porém, sua indicação não é simples e exige uma reflexão profunda, não só do ponto de vista clínico, mas também bioético. Decisões relacionadas à terapia nutricional em cuidados paliativos não são normalmente alcançadas de forma isolada; é necessário considerar o quadro clínico, os desejos dos pacientes e familiares, prognóstico, opiniões de tratamento disponíveis e seus riscos-benefícios. É fundamental estabelecer um plano de cuidado envolvendo o paciente, familiares e profissionais ${ }^{7}$.

Qual o objetivo da utilização da terapia 
Loyolla VCL, et al. Terapia nutricional enteral em pacientes oncológicos sob cuidados paliativos.

nutricional nesses pacientes? Ela traz algum benefício ou dano?

A família é esclarecida a respeito da terapia proposta? Existe realmente autonomia de decisão dos pacientes e familiares sobre a utilização da terapia nutricional?

Esses são alguns questionamentos feitos pelos familiares, cuidadores e equipe multiprofissional, que vivenciam, de alguma forma, o atendimento de pacientes oncológicos em cuidados paliativos. Questionamentos que devem ser refletidos e analisados a partir de princípios e referenciais, como beneficência, autonomia, dignidade, vulnerabilidade, entre outros, que, além de englobarem direitos e deveres, devem ser pontos de referência para a elaboração da reflexão bioética ${ }^{11}$.

Estamos diante de uma realidade complexa, na qual interferem valores e fatos de diferentes campos - cultural, profissional, religioso, científico -, que dificilmente poderá ser enfrentada com respostas simplistas e superficiais ${ }^{12}$.

Frente a este contexto, o objetivo desta pesquisa foi analisar, sob o ponto de vista da bioética, a utilização da terapia nutricional enteral em pacientes oncológicos sob cuidados paliativos, verificando a visão e a participação dos pacientes e de seus familiares na decisão sobre a utilização desta terapia.

\section{MATERIAIS E MÉTODOS}

Trata-se de uma pesquisa de campo, prospectiva, qualitativa, realizada no Hospital A. C. Camargo, em São Paulo, no ambulatório de cuidados paliativos. Foram realizadas entrevistas presenciais com pacientes portadores de câncer de cabeça e pescoço, maiores de 18 anos e seus cuidadores principais, desde que o paciente estivesse se alimentando por nutrição enteral e existisse concordância documentada do paciente e do cuidador em participar do estudo, pela assinatura do Termo de Consentimento Livre e Esclarecido. Foram entrevistados 10 pacientes e seus cuidadores principais, entre os meses de março e abril de 2010.

Os dados foram coletados por meio de um questionário semiestruturado, aplicado pelo pesquisador. Os dados gerais do sujeito de pesquisa foram organizados e apresentados por meio de tabelas, sendo analisados e comparados com referências bibliográficas e as questões abertas foram apresentadas por meio de gráficos, organizados e analisados baseados na técnica do
Discurso do Sujeito Coletivo (DSC), que, Segundo Lefèvre e Lefèvre ${ }^{13}$, em 2005, é uma técnica para fazer a coletividade falar, como se fosse um só indivíduo. Foi utilizado o software Qualiquantisoft, versão 1.3c, para o processamento dos dados.

A pesquisa foi aprovada pelo Comitê de Ética em Pesquisa do Hospital A. C. Camargo, em cumprimento com a resolução 196/96 da Comissão de Ética em Pesquisa do Conselho Nacional do Ministério da Saúde.

\section{RESULTADOS}

Participaram deste estudo 10 pacientes e seus respectivos cuidadores. A média de idade dos pacientes foi de 70,8 anos, sendo a idade mínima 60 e a máxima, 82 anos. Em relação à idade dos cuidadores, a média foi de 55,7 anos, sendo a mínima de 39 e a máxima de 82 anos.

Conforme se observa na Tabela 1, a maioria dos pacientes era do sexo masculino $(n=8)$, casada $(n=6)$, pertencente à religião católica $(n=7)$. Com relação ao nível de instrução, a categoria mais frequente foi a de primeiro grau incompleto $(n=5)$, seguida pela de segundo grau completo $(n=3)$.

Tabela 1. Distribuição da frequência dos pacientes entrevistados no ambulatório de Cuidados Paliativos do Hospital A. C. Camargo, segundo as variáveis sociodemográficas consideradas. São Paulo, 2010

\begin{tabular}{|ll}
\hline Variáveis & $\boldsymbol{n}$ \\
\hline Sexo & 10 \\
\hline Feminino & 0 \\
\hline Masculino & \\
\hline Estado civil & 8 \\
\hline Casado & 0 \\
\hline Separado & 2 \\
\hline Solteiro & 0 \\
\hline Viúvo & \\
\hline Nível de Instrução & 1 \\
\hline Ens. fund. com. & 1 \\
\hline Ens. fund. inco. & 4 \\
\hline Ens. médio com. & 0 \\
\hline Ens. médio inco. & 3 \\
\hline Superior com. & 1 \\
\hline Superior inco. & 8 \\
\hline Religião & 2 \\
\hline Católico & 0 \\
\hline Evangélico & 10 \\
\hline Espírita & 4 \\
\hline Grau de parentesco & 2 \\
\hline Cônjuge & 1 \\
\hline Filho(a) & \\
\hline Irmão(ã) & \\
\hline Nora & \\
\hline Sem parentesco & \\
\hline TOL & \\
\hline
\end{tabular}


Loyolla VCL, et al. Terapia nutricional enteral em pacientes oncológicos sob cuidados paliativos.

Referindo-se aos cuidadores, conforme a Tabela 2, verifica-se que a maioria absoluta era do sexo feminino $(n=10)$. Em relação ao estado civil, a maioria era casada $(n=8)$, e à religião, a maioria era católica $(n=8)$. Em relação ao nível de instrução, as categorias mais frequentes foram a de segundo grau completo $(n=4)$, seguida pela categoria superior completo $(n=3)$. Quanto ao grau de parentesco, a maioria dos cuidadores era cônjuge $(n=4)$, seguido por filho(a) $(n=2)$ e irmão(ã) $(n=2)$.

Tabela 2. Distribuição da frequência dos cuidadores dos pacientes entrevistados no ambulatório de Cuidados Paliativos do Hospital A. C. Camargo, segundo as variáveis sociodemográficas consideradas. São Paulo, 2010

\begin{tabular}{|c|c|}
\hline Variáveis & $n$ \\
\hline \multicolumn{2}{|l|}{ Sexo } \\
\hline Feminino & 10 \\
\hline Masculino & 0 \\
\hline \multicolumn{2}{|l|}{ Estado civil } \\
\hline Casado & 8 \\
\hline Separado & 0 \\
\hline Solteiro & 2 \\
\hline Viúvo & 0 \\
\hline \multicolumn{2}{|l|}{ Nível de Instrução } \\
\hline Ens. fund. com. & 1 \\
\hline Ens. fund. inco. & 1 \\
\hline Ens. médio com. & 4 \\
\hline Ens. médio inco. & 0 \\
\hline Superior com. & 3 \\
\hline Superior inco. & 1 \\
\hline \multicolumn{2}{|l|}{ Religião } \\
\hline Católico & 8 \\
\hline Evangélico & 2 \\
\hline Espírita & 0 \\
\hline \multicolumn{2}{|l|}{ Grau de parentesco } \\
\hline Cônjuge & 4 \\
\hline Filho(a) & 2 \\
\hline Irmão(ã) & 2 \\
\hline Nora & 1 \\
\hline Sem parentesco & 1 \\
\hline TOTAL & 10 \\
\hline
\end{tabular}

Na questão 2 do questionário aplicado, ao questionar os pacientes e cuidadores se haviam, em algum momento do tratamento, recebido da equipe multidisciplinar alguma explicação sobre a terapia nutricional enteral (alimentação por sonda), observou-se que a maioria dos pacientes $(n=9)$ e cuidadores $(n=8)$ foram informados sobre a terapia. Apenas um paciente e um cuidador relataram não ter recebido nenhuma informação e 1 cuidador não se lembrou se havia sido informado (Tabela 3).

Tabela 3. Distribuição da frequência frente à questão: "O Sr(a) recebeu alguma explicação sobre terapia nutricional enteral ou alimentação por sonda?" São Paulo, 2010

\begin{tabular}{lll}
\hline & Paciente & Cuidador \\
Sim & 9 & 8 \\
Não & 1 & 1 \\
Não lembra & 0 & 1 \\
Total & 10 & 10 \\
\hline
\end{tabular}

Referindo-se à questão 3, foi questionado qual o profissional que forneceu a explicação. Conforme mostra a Tabela 4, o nutricionista orientou a maioria do pacientes $(n=7)$ e cuidadores $(n=6)$.

Tabela 4. Distribuição da frequência frente à questão: "Em caso afirmativo, de quem recebeu esta informação?" São Paulo, 2010

\begin{tabular}{lll}
\hline Profissional & Paciente & Cuidador \\
Nutricionista & 7 & 6 \\
\hline Médico & 2 & 1 \\
Enfermeiro & 0 & 1 \\
\hline Fonoaudiólogo & 0 & 1 \\
\hline
\end{tabular}

Obs.: A mesma pessoa pode ter recebido informação de mais de um profissional.

Em relação às questões abertas realizadas nas entrevistas, os resultados obtidos são apresentados a seguir. A título de esclarecimento: DSC significa Discurso do Sujeito Coletivo, sendo A, B, C, D, E e F as categorias em que as respostas estão divididas.

Na questão 4 - Na opinião do Sr(a), o que é terapia nutricional enteral (alimentação por sonda) e para que serve? Fale um pouco sobre isso. 
Loyolla VCL, et al. Terapia nutricional enteral em pacientes oncológicos sob cuidados paliativos.

Gráfico 1. Distribuição da frequência das Ideias Centrais de cuidadores e pacientes frente à questão 4. São Paulo, 2010

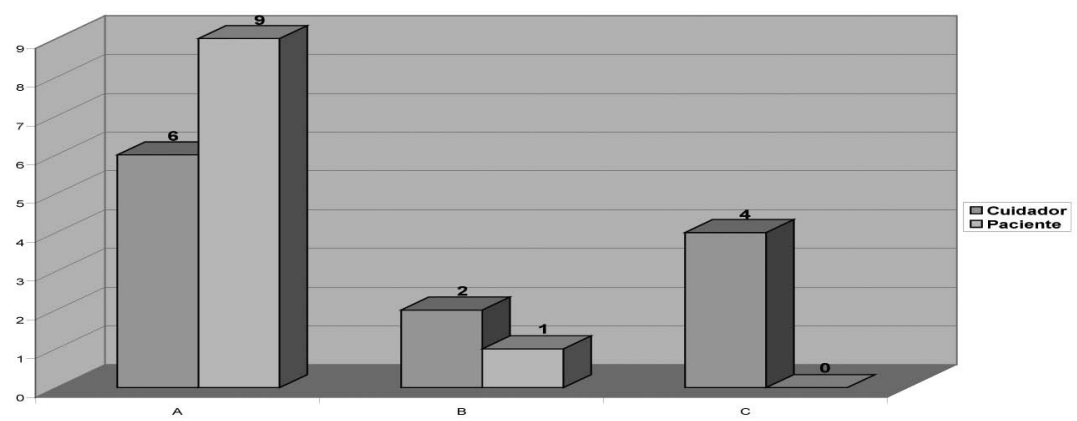

Obs.:Gráficos apresentados em números absolutos. Os entrevistados podem ter apresentado mais de uma idéia. DSC A- Serve para alimentar e fortificar o paciente. DSC B- Serve para manter / subir / recuperar o peso. DSC C- Repor, complementar as necessidades nutricionais.

Questão 5 - O Sr(a) acha que o paciente pode escolher se deve ou não iniciar a terapia nutricional enteral (alimentação por sonda)?

Gráfico 2. Distribuição da frequência das idéias centrais de cuidadores e pacientes frente à questão 5 . São Paulo, 2010

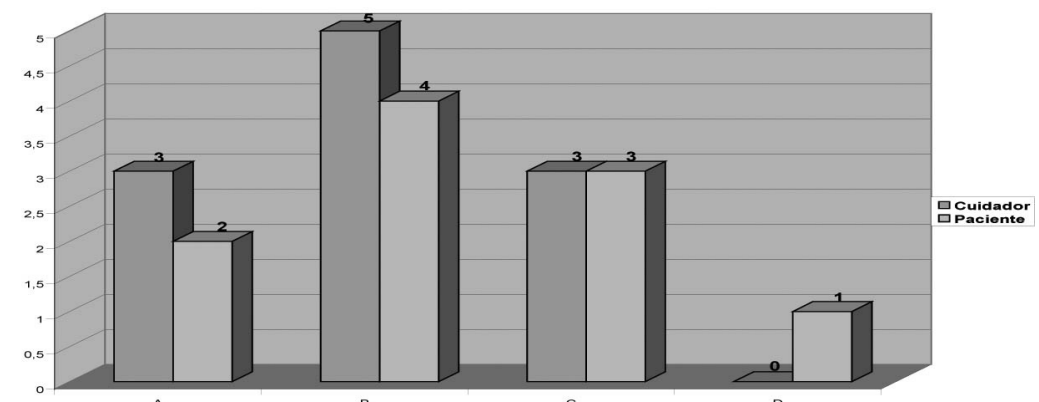

Obs.: Gráficos apresentados em números absolutos. Us entrevistados pódem ter apresentado mais de uma ideia. DSC A- Não, o paciente não pode / não deve escolher. DSC B - Sim, o paciente pode / deve escolher. DSC C - A decisão deve ser do médico. DSCD - Depende do caso.

Questão 6 - O Sr(a) acha que a família pode escolher se deve ou não iniciar a terapia nutricional enteral (alimentação por sonda)?

Gráfico 3. Distribuição da frequência das idéias centrais de cuidadores e pacientes frente à questão 6. São Paulo, 2010

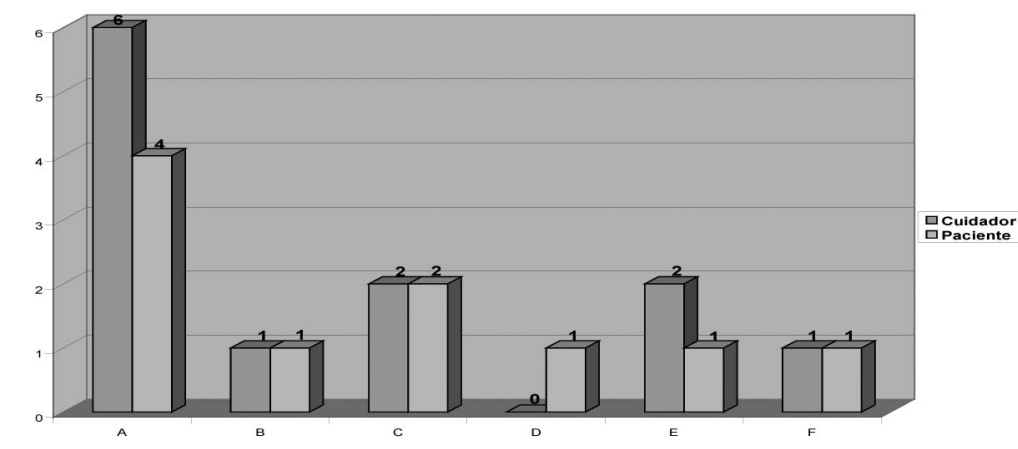

Obs.:Gráficos apresentados em números absolutos. Os entrevistados podem ter apresentado mais de uma ideia.

DSC A - Sim, se o paciente não puder escolher, a família deve decidir o que for melhor para ele. DSC B - Não, enquanto o paciente estiver lúcido. DSC C - O médico é quem deve decidir. DSC D- A família deve decidir junto com o paciente. DSC E- A família deve decidir junto com o médico. DSC F - Não sabe. 
Loyolla VCL, et al. Terapia nutricional enteral em pacientes oncológicos sob cuidados paliativos.

Questão 7 - O Sr(a) acha que a terapia nutricional enteral (alimentação por sonda) é um cuidado básico e deve ser sempre fornecido, ou é igual a um tratamento médico que pode ser interrompido caso não esteja beneficiando o paciente? Pode me explicar o porquê?

Gráfico 4. Distribuição da frequência das idéias centrais de cuidadores e pacientes frente à questão 7. São Paulo, 2010

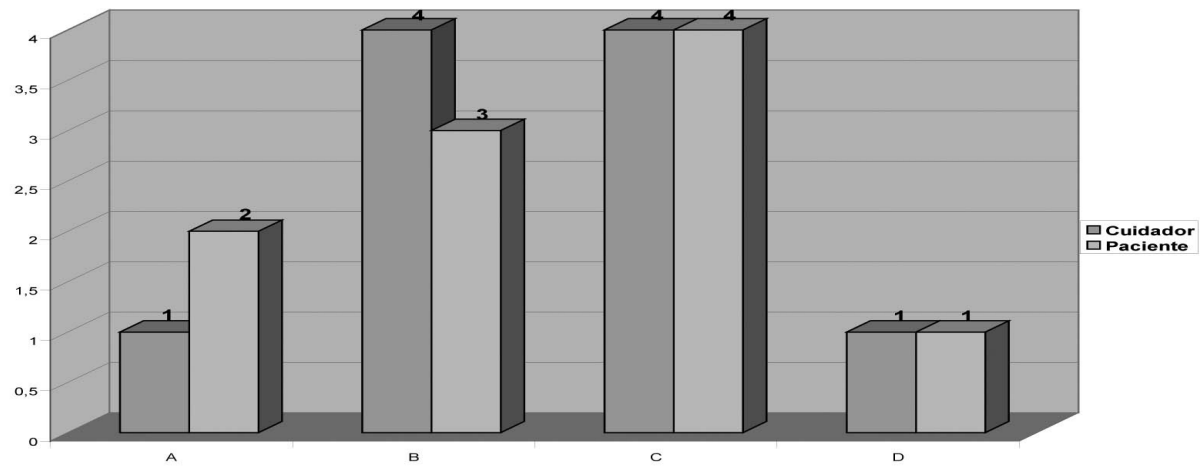

Obs.:Gráficos apresentados em números absolutos. Os entrevistados podem ter apresentado mais de uma ideia.

DSC A - Deve ser retirada caso não esteja fazendo bem. DSC B - Caso não esteja fazendo bem, deve ser substituída por outra opção. DSC C - Não pode ser retirada, pois é um direito do paciente e faz bem. DSC D - Deve ser discutido com profissional.

\section{DISCUSSÃO}

$\begin{aligned} & \text { Discussão da identificação do sujeito de } \\ & \text { pesquisa }\end{aligned}$
a do

$\mathrm{Na}$ amostra estudada, que era composta por pacientes com câncer de cabeça e pescoço, o maior número de participantes era do sexo masculino, com idade acima dos 60 anos, o que coincide com os resultados de Mendes et al. ${ }^{14}$, que, em 2006, relataram que dos 81 prontuários de pacientes ambulatoriais analisados, $63(77,8 \%)$ eram do sexo masculino, com idade média de 61,48 \pm 15,7anos.

Da mesma maneira, entre os 16 pacientes analisados por Melo et al. ${ }^{15}$ em 2006 a média de idade de era de 61,2 $\pm 12,1$ anos, sendo somente uma paciente do sexo feminino. Oliveira et al. ${ }^{16}$, em 2006, confirmam as estatísticas, pois dos 340 pacientes por eles analisados, $84,4 \%$ eram do sexo masculino e $75,0 \%$ estavam na faixa etária dos 60 anos.

Bergamasco et al. ${ }^{17}$, em 2008, no seu estudo epidemiológico do câncer de cabeça e pescoço no Estado de São Paulo, compararam a tendência do perfil epidemiológico de 16.603 pacientes no período de 2000 a 2006 e observaram que a maioria dos pacientes era do gênero masculino. No período de $2006,50,7 \%$ dos pacientes se encontravam na faixa etária de 55 a 74 anos.

Essa maior incidência no sexo masculino, na faixa etária acima dos 60 anos, pode estar relacionada ao maior índice de fumantes no sexo masculino, já que o tabaco e o álcool são reconhecidos como os mais significativos fatores de riscos associados com o desenvolvimento de carcinoma de células escamosas na cavidade oral e na faringe ${ }^{15}$.

Em relação ao nível de instrução, neste estudo, $50,0 \%(n=5)$ dos pacientes não possuem o ensino fundamental completo, resultado semelhante ao que foi observado na pesquisa realizada por Bergamasco et al. ${ }^{17}$, em 2006 na qual 46,3\% dos pacientes, no período de 2006, também não possuíam o ensino fundamental completo.

Referente ao perfil dos cuidadores encontrado, verifica-se a totalidade do sexo feminino, com a média de idade de 55,7 anos, sendo $40,0 \%$ cônjuge $(n=4)$, seguida por $20,0 \%$ de filhos e filhas $(n=2)$. Esses dados estão de acordo com os encontrados por Melo et al. ${ }^{18}$,em 2009, no seu estudo sobre o perfil social dos cuidadores de pacientes oncológicos assistidos pela equipe de Cuidados Paliativos do Sistema de Internação Domiciliar de Londrina, onde foram acompanhados 35 cuidadores, sendo $85,7 \%$ mulheres, com média de idade de 53 anos. Desses cuidadores, 37,1\% eram cônjuges e 28,6\%, filhos.

Yamashida et al. ${ }^{19}$, em 2010 com o objetivo de descrever o perfil sociodemográfico e a estrutura familiar de cuidadores de pacientes dependentes atendidos por uma equipe de saúde de uma Unidade Básica do município de São Paulo, pesquisou 67 cuidadores. Desses, 92,5\% eram do sexo feminino, com idade média de 54,7 anos. Porém, quanto ao 
Loyolla VCL, et al. Terapia nutricional enteral em pacientes oncológicos sob cuidados paliativos.

grau de parentesco, $28,4 \%$ eram filhas, $25,4 \%$, mães e 23,9\% eram cônjuges.

Os autores Silva e Acker ${ }^{20}$ em 2007, também, confirmam esses resultados, ao acompanharem oito membros de famílias que cuidavam de pacientes em cuidados paliativos domiciliar; 100,0\% eram mulheres, com as idades que variavam entre $22 \mathrm{e}$ 65 anos, sendo $75 \%$ cônjuges ou filhas.

Com relação ao nível de instrução, o presente estudo encontrou $40 \%(n=4)$ dos cuidadores com ensino médio completo e $30 \%(n=3)$ com superior completo. Melo et al. ${ }^{18}$ em 2009, em seu estudo, apresentaram um resultado semelhante, pois $34,3 \%$ dos cuidadores pesquisados apresentavam escolaridade de 9 a 11 anos de estudo.

Segundo Floriani e Schramm ${ }^{21}$ em 2004, o perfil do cuidador informal do idoso já está bem estabelecido: costuma ser do sexo feminino, filha ou esposa, muitas vezes idosa e, com frequência, divide essa atividade com seus afazeres diários.

\section{Discussão das questões 2 e 3}

Questionados quanto a terem recebido, em algum momento, informação a respeito da terapia nutricional enteral ou alimentação por sonda, a maioria dos pacientes e cuidadores respondeu afirmativamente ( $n=9$ e $n=8$, respectivamente). Este dado indica que houve a comunicação da equipe com os pacientes e cuidadores, procurando informar sobre a terapia nutricional. O profissional que mais representou essa comunicação foi o nutricionista, talvez por se tratar de um assunto específico.

É de suma importância que os médicos e os profissionais da saúde invistam um tempo para se comunicar com os pacientes, não só para explicar tecnicamente as terapias propostas, ou para dar informações sobre o tratamento, mas para ouvir os pacientes, conhecer os seus valores, suas crenças e seus objetivos. A equipe de saúde deve proporcionar as informações necessárias, de maneira simples, acessível ao paciente, para que ele possa entender as informações passadas e, no momento oportuno, tomar decisões sobre as propostas terapêuticas ${ }^{22}$.

A escolha só poderá ser feita se paciente e cuidadores forem devidamente instruídos, e, por isso, os procedimentos a serem realizados têm que ser explicados de maneira clara e compreensiva. É preciso ressaltar que, para que a autonomia seja exercida, é importante que seja reconhecida, que exista competência para tal e que de fato existam opções coerentes e satisfatórias para a tomada de decisão ${ }^{23}$.
A veracidade das informações é um dos princípios da medicina paliativa, sendo o fundamento da confiança nas relações interpessoais ${ }^{24}$. A comunicação efetiva entre médicos, equipe multidisciplinar, pacientes, familiares e cuidadores pode ajudar a prevenir dilemas éticos ${ }^{22}$.

\section{Discussão da questão 4}

As resposta à questão quatro, sobre o conhecimento que os pacientes e os cuidadores tinham a respeito do que é e da utilidade da terapia nutricional enteral, foram agrupadas em três categorias. A categoria A, "serve para alimentar e fortificar o paciente", apresentou maior frequência das respostas (6 cuidadores e 9 pacientes).

As idéias centrais das três categorias foram as respostas que os pacientes e os cuidadores deram nas entrevistas. Elas se complementaram e, de uma forma mais simples, conseguiram definir a função e a importância da terapia nutricional.

A terapia nutricional é uma modalidade de tratamento cujo objetivo é fornecer, de forma artificial, energia e nutrientes em quantidade e qualidade adequadas para suprir as necessidades diárias e para auxiliar no tratamento específico de algumas doenças; é indicada quando a ingestão oral está comprometida ou impossibilitada ${ }^{25,26}$ e visa à melhora da qualidade de vida ${ }^{8}$.

A terapia nutricional enteral foi considerada, em um dos depoimentos, como:

"substituto da alimentação convencional".

Tem o mesmo papel, a mesma importância, não só no aspecto fisiológico, como também no emocional, da alimentação oral, pois carrega uma simbologia importante para pacientes e para seus cuidadores. Pacientes e cuidadores disseram que a perda de peso e de apetite não são experiências abstratas, mas invadem e rompem a estrutura da vida diária, provocando confrontamentos, desafios sociais e questionamentos existenciais. Esses confrontamentos podem causar angústias em pacientes e cuidadores, já que não comer está relacionado ao morrer ${ }^{22,27}$.

Do ponto de vista do significado central da alimentação e da possível proximidade da morte, o alimento carrega uma simbologia significativa para pacientes e seus cuidadores. Ajudar alguém a comer pode ser um importante ato de cuidar, e, normalmente, a terapia nutricional enteral limita essa oportunidade. Os cuidadores reportaram um senso de urgência e de responsabilidade para iniciar o cuidado nutricional do paciente e demonstraram receios de que a necessidade 
Loyolla VCL, et al. Terapia nutricional enteral em pacientes oncológicos sob cuidados paliativos.

nutricional direcionasse à desnutrição $0^{22}$.

Cabe aí destacar a grande importância do acompanhamento nutricional do paciente durante todo o tratamento, avaliando suas necessidades, não só nutricionais, mas como um todo, dando suporte, também, ao cuidador, atendendo a seus anseios, respondendo às suas dúvidas e expectativas. O objetivo em cuidados paliativos não é recuperar o estado nutricional, mas proporcionar conforto, aliviar sintomas e retardar ao máximo a perda da autonomia.

\section{Discussão da questão 5}

Ao analisar a questão cinco, sobre o direito de escolha do paciente em relação ao início da terapia nutricional enteral, as idéias centrais foram agrupadas em quatro categorias distintas, sendo que a categoria que predominou foi a B (cinco cuidadores, quatro pacientes). Essa categoria deixa claro que o paciente possui o direito de escolha:

"sim, o paciente pode e deve escolher iniciar a dieta enteral".

Porém, depoimentos complementam:

"Ele tem o direito de escolher desde que esteja consciente, mas tem que ver o que é melhor para ele".

O paciente tem o direito de escolha, o direito de exercer sua autonomia. Entende-se por autonomia o direito que cada indivíduo tem de tomar decisões informadas a respeito das intervenções sobre si mesmo ${ }^{28}$.

O princípio da autonomia do paciente é a base do consentimento informado e pressupõe a necessidade de fazer um bem ao paciente. Os elementos do consentimento informado incluem informações sobre a doença, as intervenções propostas e alternativas para o tratamento, riscos e benefícios e opções de "fazer nada", entendimento das informações e acordo voluntário para as intervenções ${ }^{22}$.

Essas informações devem ser passadas pela equipe multidisciplinar, de forma clara, de maneira que o paciente entenda a informação transmitida. A equipe também necessita identificar e saber manejar os elementos da dimensão afetiva do paciente que possam interferir subjetivamente no processo de decisão ${ }^{28}$.

É de suma importância ressaltar a necessidade de se oferecer opções coerentes e satisfatórias para a tomada de decisão, para que a autonomia possa ser de fato exercida. Essas opções e procedimentos a serem realizados devem ser devidamente explicados e esclarecidos ${ }^{23}$. A autonomia somente poderá ser exercida se 0 paciente tiver capacidade de decidir; caso contrário, o cuidador deverá representar o paciente.

A capacidade para tomar decisões é a habilidade para evidenciar uma escolha, para entender e avaliar os riscos e as consequências da decisão e a habilidade para manipular racionalmente as informações ${ }^{22}$.

O paciente tem o direito de escolher, de decidir o que for melhor para ele. Mas, o que é melhor para ele? Quem sabe?

A vontade do paciente deve ser respeitada. Segundo Rodrigues e Barrera ${ }^{28}$, só procuramos o bem do paciente quando escutamos os seus desejos. Os profissionais da equipe devem despender tempo com os pacientes e encorajá-los à comunicação, para que seja possível discutir e ouvir sobre seus desejos, valores, objetivos, crenças, preferências referentes ao tratamento, sustentação da vida, enquanto esses possuem capacidade para tomar decisões ${ }^{22}$.

O mesmo deve ser feito com os familiares e cuidadores. Normalmente, as pessoas têm receio de discutir assuntos que abordem a finitude, porém essa discussão deve ser incentivada, pois é importante ao paciente expor seus desejos, vontades, angústias, receios, enquanto possui capacidade cognitiva, capacidade de decisão. Eles poderão fazê-lo utilizando declaração oral ou escrita, chamada de Declaração Antecipada, com o objetivo de orientar o tratamento no momento em que não forem mais responsáveis pelos seus atos; assim, os seus desejos se tornarão efetivos. Com isso, conflitos e dilemas éticos poderão ser evitados.

Com o novo Código de Ética Médica (CFM/ Resolução 1931/2009), artigo 37, parágrafo único, o paciente tem o direito de consentir ou recusar, de forma livre, voluntária e esclarecida, os tratamentos aos quais será submetido, pois o médico deverá considerar sua vontade expressa, ou, na sua impossibilidade, a de seu representante legal.

A segunda categoria que apresentou maior frequência nas respostas foi a C (3 cuidadores e 3 pacientes), a qual declara:

"A decisão deve ser do médico".

As justificativas foram:

"O paciente não tem capacidade para isso, é leigo. Pode até ter o livre-arbítrio, mas acaba perdendo o direito de escolha. O médico é que sabe, quem manda. A decisão é dele".

Está claro que essa relação está sendo assimétrica, desigual. A relação médico-paciente é assimétrica devido à fragilidade, natural do paciente, por um lado, e à autoridade e ao poder do profissional decorrente do saber, do outro ${ }^{29}$. 
Loyolla VCL, et al. Terapia nutricional enteral em pacientes oncológicos sob cuidados paliativos.

Ao procurar o médico, o paciente transfere a responsabilidade por si mesmo ao outro, e, muitas vezes, a atitude paternalista do médico é justificada pela busca da beneficência. O médico deve empenhar-se em fazer o bem, em proporcionar a atenção mais adequada possível ao paciente, mas se a simetria dessa relação for perdida e seus procedimentos acabarem anulando o objeto do cuidado, o paciente, essa elevação do saber ao poder levará da beneficência ao paternalismo ${ }^{29}$.

O paciente se sente leigo, incapaz de tomar a decisão, e o médico é o que sabe a verdade, pois estudou para isso. Parte da afirmativa é verdade, mas o paciente tem condições de saber o que é melhor para si. O conceito de autonomia na relação médico-paciente implica considerar que ambas as partes são capazes de decidir ${ }^{23}$.

Trindade et al. ${ }^{29}$,em 2007, entrevistaram 38 médicos responsáveis por pacientes com neoplasias malignas com o objetivo de avaliar sua postura, considerando o conflito bioético entre os princípios da beneficência e da autonomia do paciente e do paternalismo. Concluíram que o paternalismo médico surgiu com significativa presença no estudo, comprovando a dificuldade dos entrevistados de delimitar as fronteiras existentes entre autonomia do paciente e o ato beneficente do médico. A atitude paternalista emerge à medida que os recursos terapêuticos vão se esgotando. Os médicos tiveram opiniões divididas ao relacionar paternalismo e o que é fazer o bem ao paciente, revelando que muitos ainda acreditam na atitude paternalista como forma de beneficência. O estudo, no entanto, concluiu que há o início de uma postura dos profissionais no sentido de caminhar da ética médica tradicional para a Bioética.

A relação médico-paciente, no contexto de final de vida, é marcada pela impossibilidade de curar, mas o que a situação terminal traz de novo é precisamente a relativização da função curar e sua inserção numa visão mais global da interação médico-paciente. $\mathrm{O}$ limite da possibilidade terapêutica não significa o fim da relação médicopaciente. Esta ética traz à tona a essência da ética da relação ${ }^{30}$.

Em cuidados paliativos, a busca pelo ato de curar dá lugar à busca pelo ato de cuidar. A prioridade é do cuidado, não mais da cura, momento em que a dor e o sofrimento das pessoas devem ser considerados em primeira instância. O paradigma do cuidar nos permite enfrentar realisticamente os limites de nossa mortalidade e do poder médico com uma atitude de serenidade, mais participativa e simétrica. A medicina orientada para o alívio do sofrimento estará mais preocupada com a pessoa doente do que com a doença da pessoa ${ }^{30}$.

\section{Discussão da questão 6}

Em relação à questão 6 , que se propõe a averiguar se a família pode ou não escolher se deve iniciar a terapia nutricional enteral, as respostas foram apresentadas em 5 categorias, tendo a categoria A a maior frequência (6 cuidadores e 4 pacientes), trazendo a idéia central :

"Sim, se o paciente não puder escolher, a família deve decidir o que for melhor para ele".

Nesse argumento, está bem claro que o poder de decisão da família deve ser respeitado caso o paciente não tenha condições de decidir. Se o paciente não apresentar capacidade para tomar decisão, o cuidador deverá representar o paciente. Esse representante deverá ter desenvolvido uma relação de intimidade com o paciente para conhecer seus desejos, medos, preferências a fim de que a decisão a ser tomada respeite seus valores, crenças e vontades.

É fundamental ressaltar novamente a importância da comunicação entre o paciente, cuidador e equipe multiprofissional sobre assuntos referentes às terapias propostas, temas relacionados ao final de vida, quando o paciente ainda estiver em condições de se comunicar e decidir. Documentar essas decisões é importante para evitar futuros conflitos, o que pode ser feito oralmente ou com base na Diretriz Antecipada. No momento em que o cuidador tiver que tomar a decisão, deverá fazêlo considerando o ponto de vista do paciente, seus desejos, como se ele estivesse capacitado ${ }^{31}$.

Segundo Volpato e Santos ${ }^{32}$, os cuidadores, principalmente de pacientes com doenças em fase terminal, enfrentam um trabalho pesado, física e mentalmente, que pode deixá-los emocionalmente esgotados e fisicamente exaustos, completamente subjugados. Momentos de conflitos entre cuidador e paciente poderão ocorrer.

A relação entre o cuidador e o paciente dependente é complexa e, dependendo do perfil psicológico de ambos, poderá ser muito difícil, principalmente em relação à autonomia do paciente idoso, que, apesar de estar dependente e frágil, muitas vezes tem expectativa de exercitá-la. O cuidador pode erroneamente interpretar que a inabilidade de um paciente idoso para fazer algo na sua rotina seja igual à incapacidade de tomar decisões. Com isso, pode impor seu modo de realizar as atividades, não ouvindo os desejos e anseios do paciente, com erosão da autonomia e acentuação de um modelo paternalista de cuidados ${ }^{33}$.

É importante respeitar a autonomia do 
Loyolla VCL, et al. Terapia nutricional enteral em pacientes oncológicos sob cuidados paliativos.

paciente enquanto ele estiver lúcido. Este foi o depoimento de um paciente entrevistado: decide".

"enquanto o paciente estiver lúcido, é ele que

Essa resposta é referente à categoria $\mathrm{B}$. O paciente, dias depois da entrevista, foi a óbito. Foi uma comprovação real da importância de ouvir e considerar o desejo do paciente enquanto ele puder transmitir sua vontade, enquanto ele ainda estiver com capacidade para decidir.

A categoria $C$ foi a segunda que apresentou maior frequência das respostas (2 cuidadores , 2 pacientes), que diz:

"O médico é quem deve decidir".

Os argumentos aqui levantados foram os mesmos usados na questão anterior, devido à relação de paternalismo médico; uma relação assimétrica, a qual nega a liberdade de questionar a opinião médica em relação ao tratamento; uma relação em que o médico é considerado o poderoso, capaz, e o cuidador e o paciente os leigos e incapazes de decidir. Nessa relação, não se considera a autonomia de decisão nem do paciente nem dos cuidadores.

Algumas entrevistas (2 cuidadores e 1 paciente) apontaram que a família deve decidir junto com o médico (categoria E) se deve iniciar ou não a terapia nutricional enteral. Não é bem decidir, pois eles se sentem incapazes para isso, mas acompanhar, perguntar, questionar o que está acontecendo, conversar com o médico, pois eles acreditam que têm o direito. São pessoas que procuram se aproximar do médico, mas ainda de uma forma humilde, retraída, sem reconhecer seu direito de autonomia, de decisão.

\section{Discussão da questão 7}

Referente à questão 7, o objetivo foi saber se pacientes e cuidadores consideram a terapia nutricional um cuidado básico ou extraordinário. Foi perguntado se a dieta deve ser sempre fornecida, ou se pode ser interrompida caso não esteja beneficiando o paciente.

As respostas foram agrupadas em 4 categorias, sendo que as categorias $\mathrm{C}$ e $\mathrm{B}$ foram as que apresentaram maior frequência (4 cuidadores e 4 pacientes), (4 cuidadores e 3 pacientes), respectivamente.

Os depoimentos apresentados nessas duas categorias, mais os depoimentos da categoria $D$, defendem a idéia de que a terapia nutricional enteral deve sempre ser considerada um cuidado básico. Significa que, dos 20 entrevistados, 17 consideraram a terapia nutricional enteral como cuidado básico.

Na categoria C, a idéia central dos entrevistados foi:

"Não pode ser retirada, pois é um direito do paciente e faz bem".

Mesmo dizendo que se acham incapazes de opinar e decidir, eles defendem a continuidade da terapia, quando dizem:

"Não entendo como poderia ser interrompido. O que seria dado para ele? Ficar sem comer não pode, se não morre de inanição. Desde que ele começou a se alimentar está fazendo bem?'.

A alimentação, no senso de providência de comida e bebida, é parte de cuidado básico. Como comida é uma importante parte da atividade humana e cultural, muitos consideram a terapia nutricional como prática diferente de outros tratamentos médicos. As pessoas doentes devem sempre ser alimentadas quando estão com fome e poder beber quando estão com sede. Alimentar é identificado como cuidar $6,31,34$. Não se pode negar a ninguém a comida e a bebida, considerando, em qualquer caso, a alimentação e a hidratação como cuidados básicos e elementares ${ }^{35}$. É um direito do paciente e deve ser respeitado em qualquer situação.

A terapia nutricional enteral pode ser considerada um cuidado básico, mas temos que nos atentar ao fato de que, algumas vezes, essa tecnologia pode não trazer benefício. Um tratamento é benéfico se restaura a saúde, cura a doença, restaura a função, promove a comunicação ou restabelece a capacidade cognitiva e relacional ${ }^{34}$.

Em cuidados paliativos, a terapia nutricional deve aumentar a qualidade de vida, minimizar os sintomas relacionados à desnutrição e adiar ou suspender a perda de autonomia ${ }^{36}$.

"Se não estiver fazendo o bem, não pode continuar, tem que ser substituída por outra. Não pode ser retirada".

NacategoriaB, os entrevistadosconsideraram a terapia nutricional um cuidado básico, porém defenderam a idéia de que se não estiver fazendo o bem, deve ser substituída por outra opção, mas não retirada.

O acompanhamento contínuo da equipe multiprofissional para avaliar a evolução do paciente, verificar as possíveis intercorrências, a fim de adequar as terapias propostas às necessidades do pacientes, é fundamental. Se necessário, devem ser oferecidas outras opções de terapias ao paciente e ao cuidador, sempre deixando evidentes os danos que podem causar e os benefícios que podem proporcionar. 
Loyolla VCL, et al. Terapia nutricional enteral em pacientes oncológicos sob cuidados paliativos.

"Deve ser discutido com o profissionap".

Essa idéia central foi defendida pela categoria D (1 cuidador e 1 paciente), que também acredita que a terapia nutricional enteral é um cuidado básico, mas coloca no profissional a responsabilidade da decisão sobre sua continuidade se essa não estiver beneficiando o paciente. Acredita na relação de paternalismo médico, pois o "saber médico" pode garantir a beneficência ao paciente.

Temos que admitir que, algumas vezes, essa tecnologia não beneficia o paciente e, portanto, não pode continuar, tem que ser retirada. Essa foi a opinião da categoria A (1 cuidador e 2 pacientes), quando declarou: bem".

"Deve ser retirada caso não esteja fazendo

Fica claro, nesse argumento, que a terapia nutricional enteral foi considerada como medida extraordinária, igual a um tratamento médico, e deve ser suspensa caso não esteja beneficiando o paciente.

A alimentação, no senso de providência de comida e bebida, é parte de cuidado básico, mas nutrição artificial e hidratação por meio de tecnologia é um mundo à parte de comer e beber. Essa tecnologia é definida como extraordinária e, portanto, não obrigatória moralmente. Pode ser considerada como um tratamento médico, trazer benefício ao paciente ou causar danos. Alimentar uma pessoa no final de vida, quando está com fome e sede, não é o mesmo que prender a pessoa a uma tecnologia que bombeia nutrientes no trato digestório ${ }^{6,34}$.

As pessoas, quando estão iniciando o processo da morte, frequentemente, param de comer e beber. Isso é parte do próprio processo e interferir pode causar dano ao paciente ${ }^{6}$.

Determinarse uma intervençãoé moralmente extraordinária e, portanto, que não deve ser iniciada, ou que deve ser descontinuada, não é negar a pessoa do paciente ou desvalorizar a dignidade da pessoa. Não implica, necessariamente, que seja a intenção do médico ou do cuidador abreviar a vida do paciente, mas reconhecer que a saúde do paciente não poderá ser restaurada, ou que o paciente está na sua fase final e precisa ser acolhido. Procedimentos inúteis ou indesejáveis são violações à dignidade da pessoa ${ }^{6}$.

\section{CONCLUSÃO}

De acordo com os resultados obtidos, foi constatado que os pacientes e cuidadores receberam informações sobre a terapia nutricional enteral em algum momento do tratamento e que eles souberam definir sua função e importância. Porém, com a observação dos depoimentos, verifica-se que as informações transmitidas pelos profissionais não foram suficientes, ou não foram transmitidas de forma clara, para que os pacientes e cuidadores se sentissem capacitados para participar de possíveis decisões sobre a terapia nutricional enteral.

A autonomia do paciente, enquanto ele estiver consciente, em condições de decidir, deve ser respeitada. Se o paciente não possuir mais capacidade de decisão, a família deve decidir o que for melhor para ele. Essa foi a conclusão referente à autonomia de decisão.

Apesar de ter sido evidente o reconhecimento do direito da autonomia tanto dos pacientes quanto dos cuidadores na decisão referente ao início da terapia nutricional enteral, os depoimentos deixam claro que eles se consideram leigos, sentemse incapazes de discernir o que é o melhor para o paciente. Com isso, alguns deles passam a responsabilidade da decisão ao médico, com o objetivo de assegurar que a beneficência do paciente serja garantida.

A relação entre médico, paciente e cuidador ainda segue os padrões paternalistas, uma relação assimétrica, na qual o médico é que toma as decisões e paciente e cuidador se sentem incapazes para exercer sua autonomia.

Sobre a visão dos pacientes e cuidadores referente à utilização da terapia nutricional enteral, se essa é considerada um cuidado básico ou extraordinário, foi considerada pela grande maioria dos entrevistados um cuidado básico, um direito do paciente, devendo sempre ser fornecida. Caso não esteja beneficiando o paciente, deve ser substituída por outra opção, mas nunca interrompida, retirada. Os cuidadores se referem à terapia nutricional não só como algo que desempenha um papel fiosiológico, para nutrir o paciente, mas como um ato de cuidar.

Os profissionais que vivenciam o questionamento sobre a terapia nutricional enteral em pacientes oncológicos sob cuidados paliativos deve considerar cada caso clínico como um caso individual, considerando paciente e cuidador com suas características particulares, suas necessidades físicas, emocionais, espirituais, entre outras. É fundamental que haja interação entre a equipe multidisciplinar, o paciente e o cuidador, buscando sempre a melhor alternativa. Espírito de compaixão, humildade e honestidade devem estar sempre presentes. 
Loyolla VCL, et al. Terapia nutricional enteral em pacientes oncológicos sob cuidados paliativos.

Loyolla VCL, Pessino L, Bottoni A, Serrano SC, Teodoro AL, Bottoni A. Terapia nutricional enteral em pacientes oncológicos sob cuidados paliativos: uma análise da bioética. Saúde, Ética \& Justiça. 2011;16(1):47-59.

ABSTRACT: Nutritional therapy in patients with very advanced or terminal disease is frequently discussed by health care professionals. Compromised nutritional status is a common complication in cancer patients, whose weight loss is often the first symptom and is associated with increased morbidity and/or mortality. In palliative care, the primary goal of nutritional therapy is to improve the patient's quality of life, minimizing nutrition-related symptoms and postponing or avoiding loss of autonomy. Considering that bioethics is a tool for reflection and action, its principles and references must be present in every attitude and decision, which implies deep reflection by the health care team, the patient and his/her family, focusing on the patient's benefit. The aim of this study was to analyze, from a bioethical point of view, the use of enteral nutritional therapy in oncologic patients receiving palliative care, investigating participation of the patient and his/her family members in decision making regarding enteral nutritional therapy and their views on the use of this therapy.

KEY WORDS: Bioethics; Neoplasms; Hospice care; Nutrition therapy.

\section{REFERÊNCIAS}

1. Fortes PAC. Ética e saúde:questões éticas, deontológicas e legais; tomadas de decisões; autonomia e direitos do paciente; estudo de casos. São Paulo: EPU; 1998.

2. Admiraal P. Euthanasia and assisted suicidesubcortical ciscuits. In: Thomasca DC, Kushner TB. Birth to dealth. Cambridge: Cambridge; 1996.

3. Freitas GF, Fernandes MFP. Ética e moral. In: Oguisso T, Zoboli E. Ética e bioética: desafio para a enfermagem e a saúde. São Paulo: Manole; 2006. p. $45-58$.

4. Pessini L, Barchifontaine $\mathrm{CP}$, organizadores. Bioética e longevidade humana. São Paulo: Loyolla; 2006.

5 Barchifontaine CP. Bioética, cidadania e controle social. In: Pessini L, Barchifontaine CP. Bioética, longevidade humana. São Paulo: Loyolla; 2006. p .91-103.

6. Drane J, Pessini L. Bioética, medicina e tecnologia: desafios éticos na fronteira do conhecimento humano. São Paulo: Loyolla; 2005.

7. McKinlay AW. Nutrition support in patient with advanced cancer: permission to fall out? Proc Nutr Soc. 2004;633):431-5

8 Hopkins K. Food for life, love and hope: an exemplar of the philosophy of palliative care in action. Proc Nutr Soc. 2004: 63;427-9.

9 Karthaus M, Frieler F. Essen und trinken am ende des lebens. Ernahrung bei krebspatienten en der palliative onkologie und palliatinmedizin. Wien Med Wochenschr. 2004;154(9-10):192-8.

10. Wong PW, Enriquez A, Barrera R. Nutrition suport in critically ill patients with câncer. Oncol Crit Care. 2001;17(3):743-9.
11. Hossne WS. Bioética - princípios ou referenciais? Mundo Saúde. 2006;30(4):673-6.

12. Pessini L. O desenvolvimento da bioética na América Latina. In: Pessini L, Barchifontaine CP. Fundamentos da bioética. São Paulo: Paulus; 1996. p.9-29.

13. Lefèvre $F$, Lefèvre $A M$. $O$ discurso do sujeito coletivo: um enfoque em pesquisa qualitativa. Caxias do Sul: EDUCS; 2005.

14. Mendes CCT, et al. Avaliação do estado nutricional de pacientes com câncer de cabeça e pescoço em acompanhamento ambulatorial. Rev Bras Nutr Clin. 2006;27(1):23-7.

15. Melo ILPM, Dantas MAM, Silva LC, Lima VT, Lima SCV, Sena KCM. Avaliação nutricional de pacientes cirúrgicos com câncer de cabeça e pescoço sob terapia nutrição enteral. Rev Bras Nutr Clin. 2006;1(1):6-11.

16. Oliveira LR, Ribeiro-Silva A, Zucoloto S. Perfil da incidência e da sobrevida de pacientes com carcinoma epidermóide oral em uma população brasileira. J Bras Patol Med Lab. 2006;42(5):38592.

17. Bergamasco VD, Marta GN, Kowalski LP, Carvalho AL. Perfil epidemiológico do câncer de cabeça e pescoço no Estado de São Paulo. Rev Bras Cir Cabeça Pescoço. 2008;37(1):15-9.

18. Melo TM, Rodrigues IG, Schmidt C. Caracterização dos cuidadores de pacientes em cuidados paliativos no domicílio. Rev Bras Cancerol. 2009;55(4):36574.

19. Yamashida $\mathrm{CH}$, Amendola $\mathrm{F}$, Alvarenga MRM, Oliveira MAC. Perfil sociodemográfico de cuidadores familiares de pacientes dependentes atendidos por uma unidade de saúde da família no município de 
Loyolla VCL, et al. Terapia nutricional enteral em pacientes oncológicos sob cuidados paliativos.

São Paulo. Mundo Saúde. 2010;34(1):20-4.

20. Silva CAM, Acker JIBV. O cuidado paliativo domiciliar sob a ótica de familiares responsáveis pela pessoa portadora de neoplasia. Rev Bras Enferm. 2007;60(2):150-4.

21. Floriani AC, Schramm FR. Atendimento domiciliar ao idoso: problema ou solução. Cad Saúde Pública. 2004;20(4):986-94.

22. McMahon MM, Hurley D, Kamath PS, Mueller PS.Medical and ethical aspect of long-term enteral tube feeding. Mayo Clin Proc Rochester. 2005;80(11):1461-76.

23. Kòvacs MJ. Comunicação nos programas de cuidados paliativos: uma abordagem multidisciplinar. In: Pessini L, Bertachini L, organizadores. Humanização e cuidados paliativos. São Paulo: Loyola; 2004. p. 275-86.

24. Pessini L. Distanásia: até quando prolongar a vida? São Paulo: Loyola; 2001.

25. Garofolo A. Diretrizes para terapia nutricional em crianças com câncer em situação crítica. Rev Nutr. 2005;18(4):513-27.

26. Caruso L, Simony RF, Silva ALND. Dietas hospitalares: uma abordagem na prática clínica. São Paulo: Atheneu; 2004.

27. Strasser F, Binswanger J, Cerny T. Fighting a losing battle: eating related distress of men with advanced cancer and their female partners. A mixed methods study. Palliative Med. 2007;21(2):129-37.
28. Rodrigues T, Barrera RL. Analisis del problemas éticos frecuentes en medicina paliativa. ARS Med (Santiago). 2005;11(11):43-56.

29. Trindade ES, Azambuja LOA, Andrade JP, Garrafa V. O médico frente ao diagnóstico e progóstico do câncer avançado. Rev Assoc Med Bras. 2007;53(1):68-74.

30. Pessini L, Bertachini L, organizadores. Humanização e cuidados paliativos. São Paulo: Loyola; 2004.

31. Hook CC, Mueller OS. The Terri Schiavo saga: the making of tragedy and lesson learned. Mayo Clin Proc. 2005;80(11):1449-60.

32. Volpato FS, Santos GRS. Pacientes oncológicos: um olhar sobre as dificuldades vivenciadas pelos familiares cuidadores. Imaginário. 2007;13(14):51144.

33. Neri AL. Bem- estar e estresse em familiares que cuidam de idosos fragilizados e de alta dependência. In: Liberalesso A. Qualidade de vida e idade madura. Campinas: Papirus; 2000. p. 237-85.

34. Drane JF. Stopping nutrition and hydration technologies: a conflict between traditional catholic ethics and church authority. Christ Bioeth. 2006;12(1):11-28.

35. Fernandez-Rondán AC. Nutritión en el paciente terminale. Punto de vista ético. Nutr Hosp. 2005;20(2):88-92.

36. Caro MMM, Laviano A, Pichard C. Nutritional intervention and quality of life in adult oncology patients. Clin Nutr Metabol. 2007;26:286-301.

Recebido em: 21/12/2010

Aprovado em: 28/01/2011 